\title{
In Memoriam Diego Robles Marin (1931-1992)
}

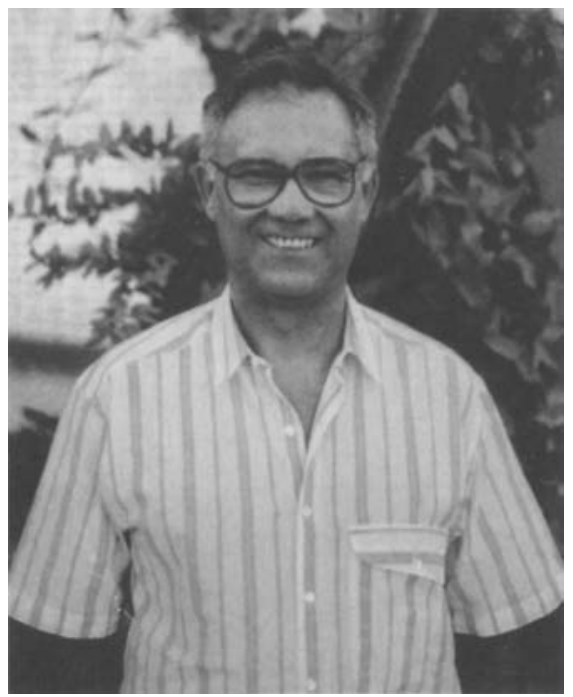

Le nom de Diego Robles Marin, un prestigieux orthopédiste de la communauté valencienne, est associé sans aucun doute à ses nombreuses investigations tant dans les domaines de la Traumatologie et de l'Orthopédie, que de l'Anatomie et la Biomécanique de 1'Appareil locomoteur. Ce grand travail commença en 1968 et a fructifié dans de nombreuses Thèses doctorales, des publications dans des revues nationales et internationales, symposiums et réunions, qui culminèrent en 1986 avec la fondation de la Société Espagnole d'Investigation de la Colonne Vertébrale (SEICV) et la création de l'Unité du Rachis Expérimental (URE) à l'Université de Valencia. Sans vouloir omettre la grande variété de ses travaux, sa plus grande préoccupation comme médecin et chercheur fut en effet le Rachis. Tous ses mérites sont soulignés par sa grande humanité qui a toujours rejailli sur les nombreux élèves qu'il a formés.

Il naquit en 1931 à San Sébastian bien que sa famille vécut à Calahorra, où il passa son enfance et son adolescence pendant les dures années de la Guerre Civile espagnole. Quelques années plus tard, sa famille se fixa à Valencia où il commença ses études de Médecine dans la vieille Faculté, pour les terminer en 1954.

En 1959 et 1960, il est résident à l'Hôpital Leuval de Nice, sous la direction du Professeur Descamps. En 1961, il travaille au Pavillon Ollier de l'Hôpital de Cochin, qui vient d'être inauguré, comme médecin-assistant du Professeur Robert Merle d'Aubigné. De ce séjour naquit une profonde amitié qui alla au delà de la seule relation maître-élève et qui se maintiendra jusqu'à la mort de ce dernier en 1989. C'est avec ces célèbres maîtres que naquit sa vocation pour la Traumatologie et l'Orthopédie.

De retour à Valencia, il obtint en 1964 le titre de Spécialiste en Orthopédie-Traumatologie qu'il exercera tant en public qu'en privé, imitant en cela le modèle français de l'assistance intégrée au malade.

En 1968, il commence ses études et recherches sur le colonne vertébrale au Département d'Anatomie de l'Université de Valencia, sous l'orientation du Professeur Victor Smith Agreda. Sa thèse sur la vascularisation de la colonne vertébrale est soutenue trois années plus tard. En 1972, il est nommé Professeur Adjoint et peut alors se consacrer tant à l'enseignement qu'à la recherche sur la colonne vertébrale.

En 1973, il est accepté comme membre par les Sociétés Espagnole et Française d'Orthopédie et par la Société Espagnole de Microscopie Electronique. En 1975, il reçoit le Prix de l'Académie Royale de Médecine de Valencia pour son travail sur l'ossifica- tion de la colonne vertébrale et devient membre de cette prestigieuse Académie. La même année il devient membre de la Société Internationale de Chirurgie Orthopédique et Traumatologique, grâce à ses contributions dans ces domaines.

Pendant ce temps de nombreuses présentations et articles dans des congrès nationaux et internationaux font état de ses travaux, sans oublier son travail quotidien auprès de ses patients. En 1981, il est nommé membre de la Société Ibérique de Biomécanique grâce à ses études sur le pelvis et le rachis et son diplôme d'études avancées à l'Ecole Supérieure d'Ingénieurs de l'Université Polytechnique de Valencia.

En 1983 il obtient la place de Professeur Titulaire du Département d'Anatomie de la Faculté de Médecine de Valencia. Il va alors cristalliser ses préférences scientifiques en créant en 1986 la SEICV et l'URE, sociétés dont il fut président et directeur. En 1987, il est nommé membre de la branche européenne de la Cervical Spine Research Society dont il est, avec le Professeur Canadell l'un des deux membres espagnols.

Diego Robles Marin n'a pas seulement été pour ses disciples un authentique maître, il a surtout su être un ami et un collègue avec des lettres majuscules. Même s'il nous a quittés ce 18 janvier 1992, l'esprit de son oeuvre bat avec une force égale, sinon peut être plus grande, dans le coeur de tous ceux qui eurent la chance d'apprendre avec lui.

Repose en paix. 